\title{
Measuring the underwater received power behavior for 433 mhz radio frequency based on different distance and depth for the development of an underwater wireless sensor network
}

Muhammad Ramdhan M. S., Muhammad Ali, Nurzal Effiyana G., Samura Ali, Kamaludin M. Y. Advanced Telecommunications Technologies (ATT) Research Group, Communication Department, Faculty of Electrical Engineering, Universiti Teknologi Malaysia, 81310 Johor Bahru, Malaysia

\begin{tabular}{l} 
Article Info \\
\hline Article history: \\
Received Dec 29, 2018 \\
Revised Mar 6, 2019 \\
Accepted Mar 29, 2019 \\
\hline
\end{tabular}

Keywords:

Internet of Things (IoT)

Radio Frequency (RF)

Underwater Wireless Sensor

Network (UWSN)

\begin{abstract}
Underwater wireless sensor network (UWSN) important to enhance the widely use of the application of the Internet of things (IoT) for underwater. Uses of the acoustics base of wave propagations are the best ways to establish the UWSN. But the unpracticality of the hardware due to the size and cost has limited the application of UWSN. Radio frequency (RF) wave propagation is the best way to overcome this situation. Low frequency of the RF wave is proven feasible and suitable for underwater communication. 433 $\mathrm{MHz}$ RF were chosen to measuring the underwater received power behavior between the transmitter node and receiver node based on different distance and depth. HC12 transceiver module was used as a transmitter and spectrum analyzer with the telescopic antenna was used as a receiver. The received power give a good reading when the transmitter note was at 0.5 -meter depth with a maximum operating range within 12 meters from the receiver.
\end{abstract}

Copyright $(2019$ Institute of Advanced Engineering and Science. All rights reserved.

\section{Corresponding Author:}

Muhammad Ramdhan M. S.,

Advanced Telecommunications Technology Research Group,

Faculty of Electrical Engineering, Universiti Teknologi Malaysia (UTM),

Skudai, 81310, Johor, Malaysia.

Email: mramdhan2@live.utm.my

\section{INTRODUCTION}

The expansion of internet connectivity nowadays gives people unlimited access to the virtual network at anytime and anywhere [1]. Therefore, the Internet of Things (IoT) has gained rapid attention from the researchers due to the expansion of the internet [2]. The quality and efficiency of human daily life have improved driven by the implementation of IoT in daily life [2]. The implementation of a Wireless Sensor Network (WSN) will give a lot of advantages in IoT applications in term of monitoring and control. WSN is a wireless network consisting of spatially distributed autonomous devices knows as sensors nodes to monitor physical or environmental conditions [3]. These technologies have been deploys in many sectors such as agricultural, ecological, safety, sport, transportation and healthcare. The rising of the WSN development are due to low cost and ease of deployment in any types of applications [4]. Implementation of the underwater WSN has change landscape of IoT. Normal WSN architecture was using air as a medium for transmitting data. Thus, the IoT application is widely developed for surface application compare to the underwater application. The Underwater Wireless Sensor Network (UWSN) are the other extended division of WSN and it extremely challenging [5].

When talking about underwater communications, acoustics propagation is more convenient compared to RF propagation and become favorable among researchers. However, acoustics propagation has its own drawback. Acoustics wave propagation is not considered suitable for underwater real-time applications [5]. It is due to the underwater acoustics suffer from its low velocity, and the operating frequencies limit the bandwidth due to the multipath and Doppler Effect [6]. Other than that, underwater 
acoustic attenuation is unfeasibly high in shallow water application [7]. Besides, underwater acoustics suffer poor performance due to turbidity and ambient noise [8]. For sizing and cost, acoustic propagation uses a large piece of hardware and it expensive [9].

In another hand, RF communication has been very unfavorable for underwater communication due to high attenuation and it needs to deploy more nodes to transmit and relay the signal to the based station [10-12]. However, RF has been acknowledged as a good alternative for replacing the acoustic type of wave propagation in UWSN [13]. The RF propagation velocity is much faster than the acoustic wave, up to $3 \times 107 \mathrm{~m} / \mathrm{s}$, therefore, the latency of wave propagation can be reduced [14]. Besides that, RF wave offers many advantages over acoustic propagation including wider bandwidth, high data rate and better transmission in crossing shallow water since it can tolerate to ambient noise $[8,14,15]$.

Since the evolution of WSN technology is increasing, there a lot of transceivers are developed such as NRF24101, Xbee and many more. This paper was discussed the received power behavior for $433 \mathrm{MHz}$ underwater RF based on the variances depth and distance between the transmitter node and the receiver node. HC12 transceiver module was used due to the small size, low power consumption and better range for wave propagation and the module are already existing in the market [16]. $433 \mathrm{MHz} \mathrm{RF}$ was chosen due to the low attenuation value compare to other frequency on another transceiver module [17]. In this paper, $433 \mathrm{MHz} \mathrm{RF}$ wave propagation will be outlined and the theory will be reviewed. This will be used to prove in theory and practice that $433 \mathrm{MHz}$ RF propagation is feasible at a certain depth and a certain distance between transmitter and receiver node. It will prove that the RF wave might be used for real-time underwater WSN applications in solution for underwater monitoring and safety purposes.

\section{RESEARCH THEORY}

The received power is an important parameter in wireless telecommunication. Received power will show the overall performances of the RF. Received power equations are shows as Friss (1) [18] below.

$$
(d B m)=P_{\mathrm{t}}(d B m)+G_{t}(d B)+G_{r}(d B)-L_{\text {pathloss }}(d B)
$$

where; $\mathrm{P}_{\mathrm{t}}=$ Transmit power

$\mathrm{G}_{\mathrm{r}}=$ Gains of the receiver antenna

$\mathrm{G}_{\mathrm{t}}=$ Gains of the transmitter antenna

$L_{\text {Pathloss }}=$ Path loss in water

The range of RF was based on how high the path loss values. It depends on the communication medium that the RF need to go through. From (1), the power received will give a lower reading as the path loss of underwater RF propagation is high. It may be due to many factors such as refraction, diffraction, reflection, absorption of water and the distance between the transmitter and the receiver. All of these factors were depends on the water characteristics, which is permeability $\mu$, conductivity $\sigma$, density $\rho$ and permittivity $\varepsilon[19]$.

These characteristics will cause the propagation speed for underwater communication slow by a factor of 9 compared to propagation speed for free space. Underwater propagation speed can be expressed as (2) [20].

$$
c \approx \frac{1}{\sqrt{\varepsilon \mu}}
$$

Besides, the absorption coefficient, $\alpha$ for RF propagation underwater is also higher compare to the absorption coefficient for free space. It can be expressed as (3) [20].

$$
\alpha \approx \frac{\sigma}{2} \sqrt{\frac{\mu}{\varepsilon}}
$$

Propagation speed and absorption coefficient will cause the power received to decrease as the distance and depth between the transmitter and receiver node are increase.

\section{RESEARCH METHODOLOGY}

To analyze the behavior of the power received at the receiver, all the experiments were conducted in the same and control environment. These experiments were conducted at the swimming pool that belongs to Universiti Teknologi Malaysia, Johor Bahru. The swimming pool size is 20 meter x 25 meter x 1.5 meter with the same type and volume of water. The experiments were conducted during a calm water situation. 
(Note: The experiment conducted in a safe environment under the supervision of the accredited UTM swimming pool lifeguard). The idea is the transmitter node transmits the packets by using the broadcasting data transmissions method to the receiver node located close to the swimming pool. The spectrum analyzer was acted as a receiver node that shows the received power in $\mathrm{dBm}$. The collected data will be in the form of variables, received power in $\mathrm{dBm}$ and sets of the coordinates for all the measured locations. Figure 1 shows swimming pool at Universiti Teknologi Malaysia, Johor Bahru.

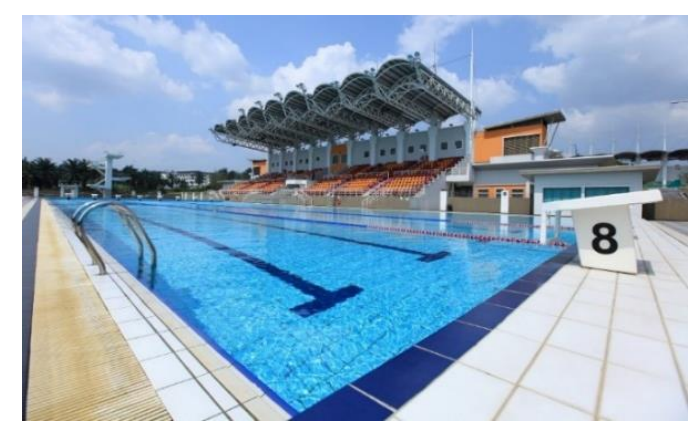

Figure 1. Swimming pool at Universiti Teknologi Malaysia, Johor Bahru

\subsection{Hardware configurations}

For the hardware, there are two main nodes were used for this experiment, the transmitter node and the receiver node. Each of the nodes has its own setup that needs to prepare before carrying out this experiment. All of this setup configuration shown below.

\subsubsection{Transmitter}

For this experiment, the transmitter node is made up of a few components that embedded together. The Arduino Pro Mini will act as a microcontroller for this transmitter node due to its suitability for the transmitter node design. Even though the size for this Arduino pro mini is small, it has a large memory and has the ability to communicate with $\mathrm{HC} 12$ transceiver module through its own built-in serial port (RXD and TXD pins) [21]. The 9V battery is act as the input voltage for the circuit. Figure 2 shows the full schematics diagram for the transmitter node.

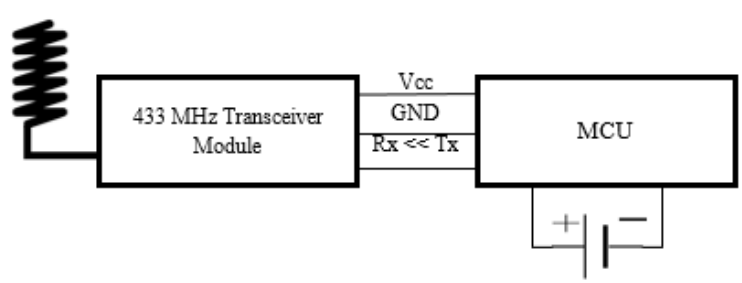

Figure 2. Transmitter node schematics diagram

In this experiment, HC12 Transceiver Module was set to channel 1 via AT command with a center frequency of 433.4 MHz and default working mode (FU3; full-speed mode) with 9600 bps (serial port baud rate) and 15000 bps (baud rate in the air). This experiment only involved half-duplex communication only. Therefore, only the Tx pin on the microcontroller is connected to the Rx pin on the HC12 transceiver module. The packet size for this experiment was only 9 bits (data size: 8 bits, Stop bit: 1 bit). To make it waterproof, the transmitter circuit were sealed inside the plastic box. Figure 3 shows packet size.

\section{Payload (9 bits) $\quad$ Stop bit (1 bit)}

Figure 3. Packet size 
Transmitter node is using $27 \mathrm{~mm}$ coil spring antenna types of antenna that made up from phosphorus copper material with the standing-wave ratio (SWR) less than 2.0. It has 50 ohms impedance with a gain of $2.0 \mathrm{dBi}$. Transmit power for this transmitter node is $13 \mathrm{dBm}$. It used GFSK types of modulation since Si4464 transceiver chip from Silicon Lab were build in inside the HC12 transceiver module [22]. It has a 100 communicational channel with $400 \mathrm{kHz}$ bandwidth each channel [22]. The transmitter node was used wireless Universal Asynchronous Receiver/Transmitter (UART) protocol for transmitting the packet [22].

\subsubsection{Receiver}

For the receiver node, portable Keysight N9342C Handheld Spectrum Analyzer (HSA) acted as receiver node. It can measure the frequency up to $7 \mathrm{GHz}$, therefore it is suitable for measuring the received power for this experimental $433 \mathrm{MHz}$ [23]. ANT500 telescopic antenna is connected to the spectrum analyzer through 1 meter of coaxial cable. The antenna has a range of operating frequency starting $75 \mathrm{MHz}$ to $1 \mathrm{GHz}$ with $50 \mathrm{ohms}$ of impedance and $0 \mathrm{dBi}$ of gain [24]. To run this experiment, the spectrum analyzer setup is like in Table 1.

Table 1. Spectrum analyzer settings

\begin{tabular}{cc}
\hline Parameter & Value \\
\hline Impedance & $50 \mathrm{Ohm}$ \\
Start Frequency & $+4.134000000 \mathrm{e}+08 \mathrm{~Hz}$ \\
Stop Frequency & $+4.534000000 \mathrm{e}+08 \mathrm{~Hz}$ \\
Frequency Offset & $+0.000000000 \mathrm{e}+00 \mathrm{~Hz}$ \\
Bandwidth & $40 \mathrm{MHz}$ \\
Center Frequency & $433.4 \mathrm{MHz}$ \\
Attenuation & $10 \mathrm{~dB}(\mathrm{AUTO})$ \\
Resolution Bandwidth (RBW) & $300 \mathrm{z}$ \\
\hline
\end{tabular}

\subsection{Experimental setup}

Figure 4 and 5 shows the experimental setup for this experiment. This experiment measured the received power between the transmitter node and receiver node at a different distance starting from 0 meter to 14 meter with a 2 meter increment for each reading. The measurement also carried out at varies of depth starting 0 meter to 1.5 meter with a 0.5 meter increment for each reading. The position of the transmitter node location is determined by using meter tape. Therefore, there are 32 average mean data for each location. Every point of measurement ware taking takes 10 readings, therefore there is 320 data were taken during the experiment. For capturing the data, the max hold application method was used [25]. The telescopic antenna for the receiver was placed at the $(0,0)$ coordinate and this location is fixed until the end of the experiment. Figure 6 shows side view of the swimming pool for the experimental setup.

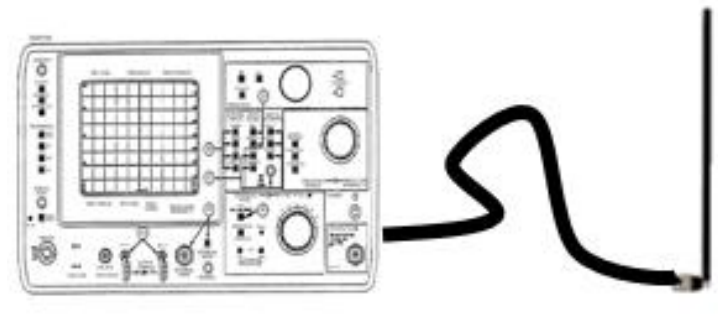

Figure 4. The setup connection of the receiver node

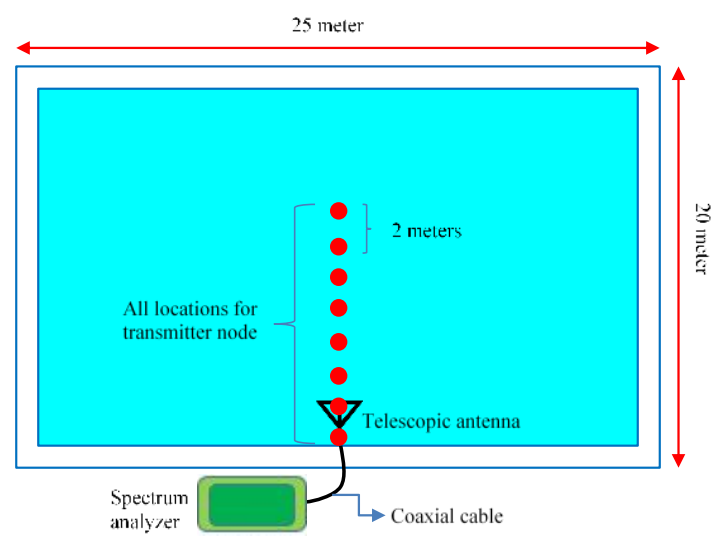

Figure 5. Top view of the swimming pool for the experimental setup 


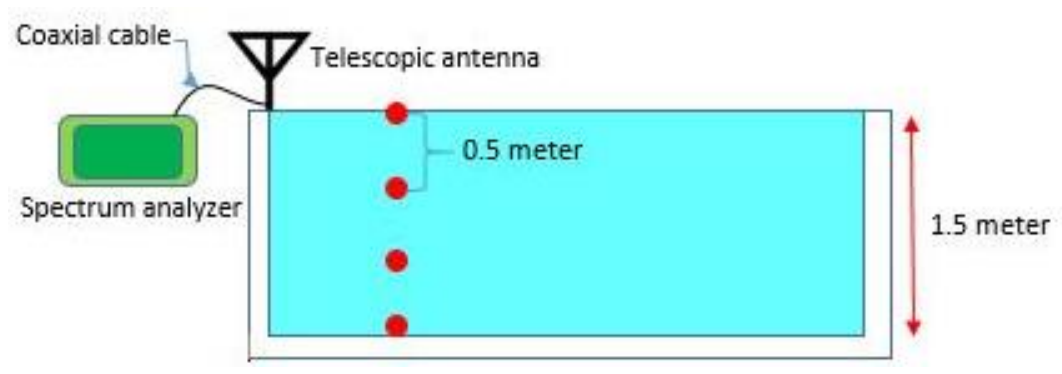

Figure 6. Side view of the swimming pool for the experimental setup

Before the experiments begin, the noise level was measured. Figure 7 shows the noise floor level at the UTM swimming pool during the experiment were conducted. The average value for measurements, data1 and data 2 is $-82.0551 \mathrm{dBm}$ and $-81.6926 \mathrm{dBm}$. Thus, the overall mean for the noise level is $-81.8738 \mathrm{dBm}$.

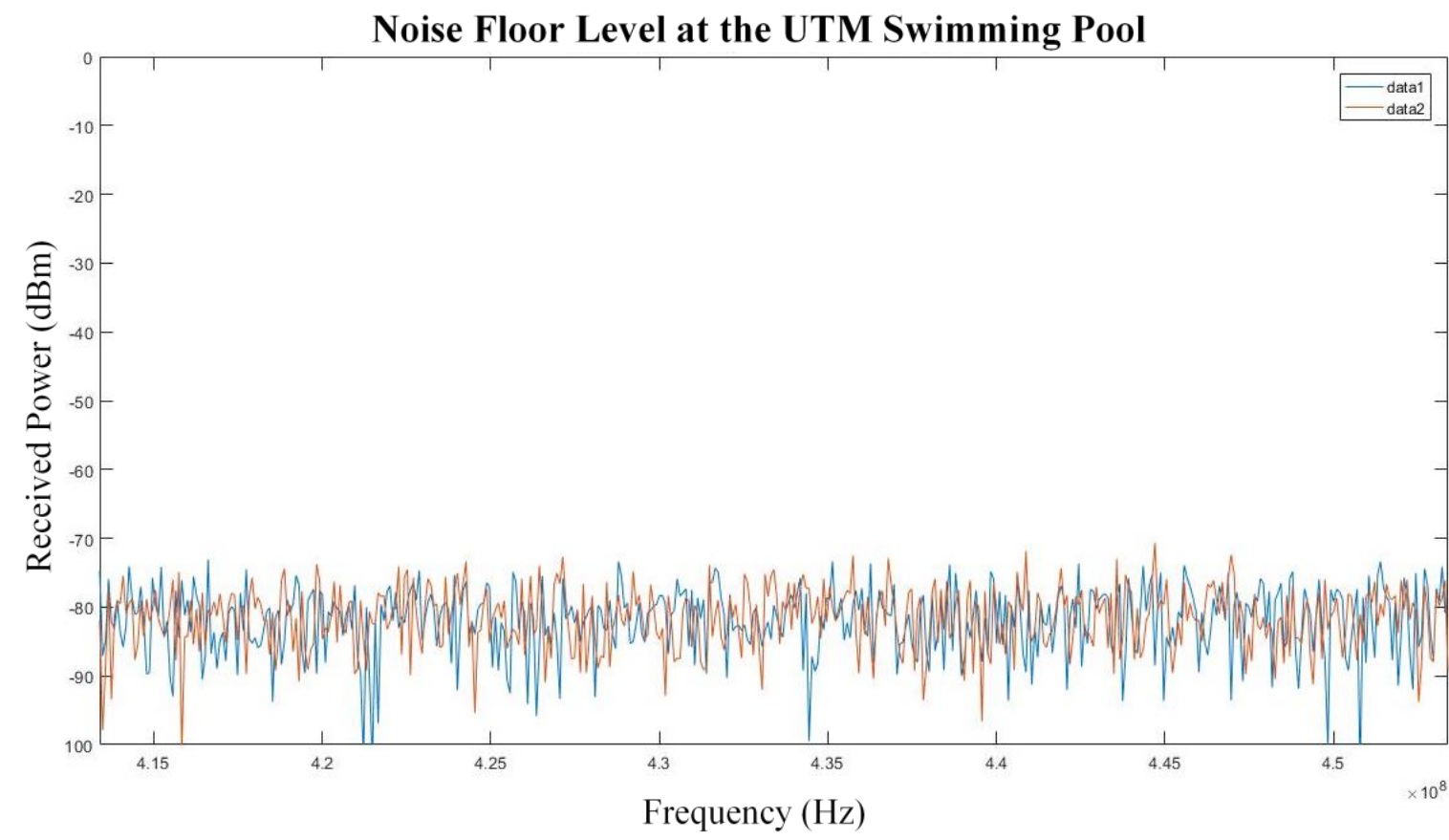

Figure 7. Noise Floor level for two measurements

\section{RESULTS AND ANALYSIS}

Table 2 shows the result of the experiment. This table represents the average mean of 10 readings for each location. Total location for the transmitter node located inside the swimming pool is 32 locations. From Table 2, the maximum amplitude for the received power is $-57.8 \mathrm{dBm}$ when the transmitter node was located at coordinate $(0.0 \mathrm{~m}, 0.0 \mathrm{~m})$. It is due to the location between these two node is near to each other. Therefore, the loss at this coordinate is minimal when comparing it to other locations. The minimum amplitude for the received power $-83.7 \mathrm{dBm}$ when the transmitter node was located at coordinate $(4.0 \mathrm{~m}, 1.5$ $\mathrm{m}$ ) which means that the transmitter node was at the bottom of the swimming pool that located 4 meters away from the receiver node. Since the value for received power at $(4.0 \mathrm{~m} .1 .5 \mathrm{~m})$ is $-87.3 \mathrm{dBm}$, the value has already succeeded the value for average noise floor value, the packet data were stopped receive at the receiver node at this point. 
Table 2. Average for mean for received power in $\mathrm{dBm}$ at $0.0 \mathrm{~m}, 2.0 \mathrm{~m}, 4.0 \mathrm{~m}, 6.0 \mathrm{~m}, 8.0 \mathrm{~m}, 10.0 \mathrm{~m}, 12.0 \mathrm{~m}$ and $14.0 \mathrm{~m}$ based on the different depth of $0 \mathrm{~m}, 0.5 \mathrm{~m}, 1.0 \mathrm{~m}$ and $1.5 \mathrm{~m}$

\begin{tabular}{lllllllll}
\hline & \multicolumn{7}{c}{ Distances } \\
\hline $0.0 \mathrm{~m}$ & -50.8 & -59.5 & -58.3 & -62.6 & -64.9 & -63.2 & -69.4 & -74.2 \\
$0.5 \mathrm{~m}$ & -58.6 & -67.6 & -65.5 & -73.1 & -73.8 & -72.1 & -82.2 & -82.1 \\
$1.0 \mathrm{~m}$ & -66.1 & -76.3 & -82.1 & -82.7 & -83.1 & -82.4 & -82.3 & -83.2 \\
$1.5 \mathrm{~m}$ & -70.3 & -77.1 & -83.7 & -82.3 & -82.4 & -82.3 & -83.3 & -83.0 \\
\hline
\end{tabular}

Theoretically, the received power is continuously decreasing as the distance between the transmitter and the receiver node is increased. The decreasing of the received power amplitude supposedly in uniform or nearly uniform as the distance between these two nodes is increased. But, in this experiment, it shows that the received power is not decreased uniformly. Based on Figure 8, when the transmitter node was at the surface of the swimming pool (depth=0.0 meter), the amplitude for received power is not constantly decreasing. It is due to the loss due to the reflection and refraction of RF wave with the water. The packet data is still received when the transmitter node at the farthest point of 14 meter. When moving to the depth of 0.5 meter, the power received reached the noise level at 12 meter and it became worst when it moves to the depth of 1.0 meter and 1.5 meter. The packet data can only be transmitted until less than 4 meter for 1.0-meter depth and 1.5-meter depth. The graph in Figure 8, shows that the average of the noise floor for this experimental situation. During this experiment, the average noise floor reading is $-81.8738 \mathrm{dBm}$. On this noise floor level, the packet data were no longer received by the receiver due to the dynamic range between the received power and noise floor level is very small.

Power Received (dBm) for All Locations of Transmitter Node

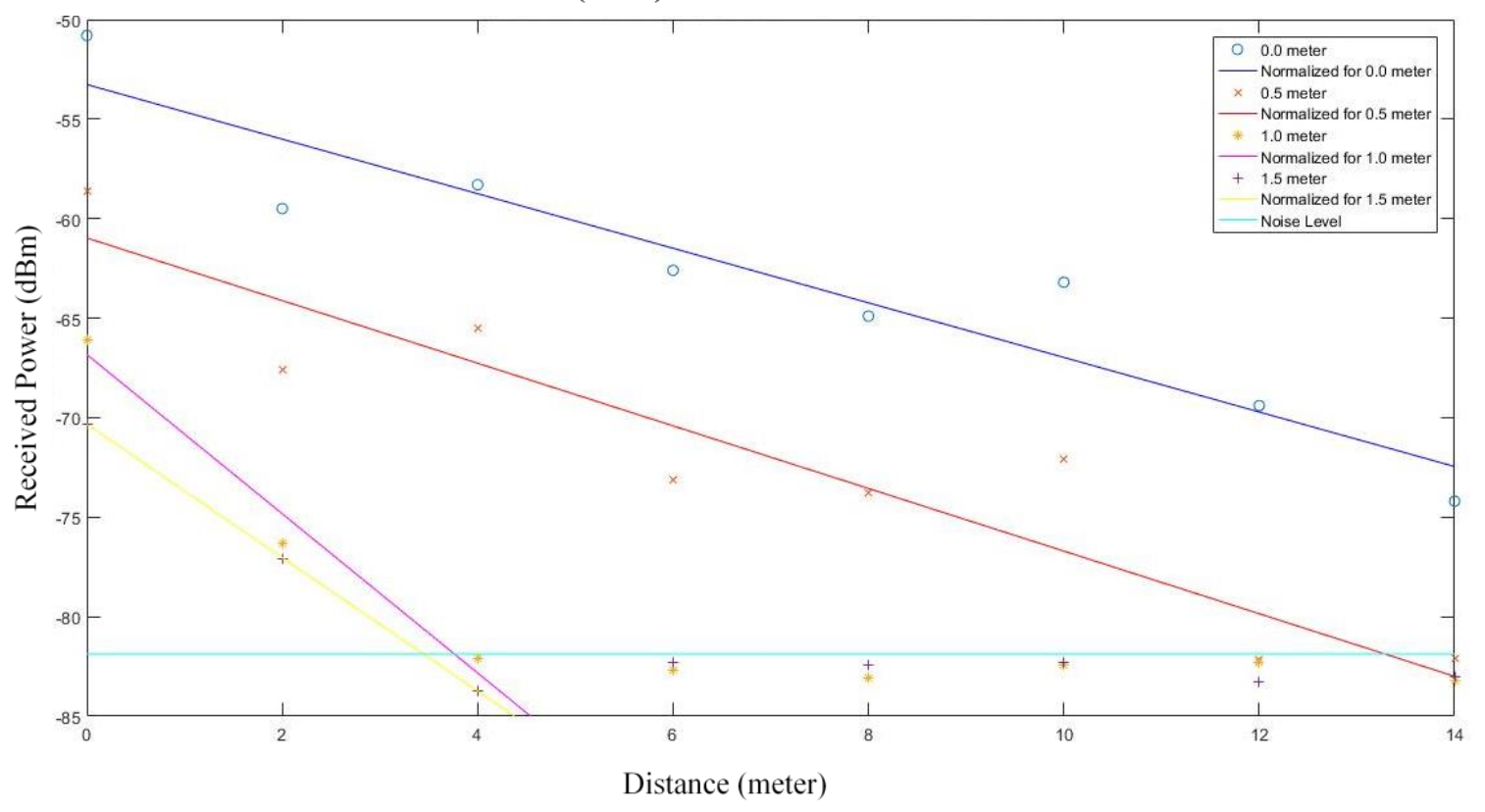

Figure 8. Received Power Result based on the different distance between transmitter and receiver

Figure 8 shows the received power behavior for $433 \mathrm{MHz}$. It can be shown from 4 different graphs which based on the 4 different depth during the experiment, these graphs are the normalized reading from all the locations. All of these graphs have their own characteristics. All of this graph is linear but it is not identical to each other. Every graph has a different gradient value, therefore the rate of received power depletion are also different. The deeper the transmitter node located, the higher the value of the gradient, thus the rate of power received depletion also will increase as we increase the distance between the transmitter node and the receiver node. While the transmitter node at the surface of the swimming pool; depth $=0.0$ meter, the power received has depleted $1.375 \mathrm{dBm}$ per meter as it moved from 0 meter to 14 -meter distance from the receiver node. When the transmitter was at level 0.5 meter underwater, the signal has completely lost 
when it reaches 12 meter distance from the receiver node. For these conditions, the power received were depleted $1.6 \mathrm{dBm}$ per meter. As it moved deeper to 1.0 meter of depth, the received signal power has lost 4 $\mathrm{dBm}$ per meter and it the signal only can be reached until 4-meter distance from the receiver node. The situation is the same when the transmitter node was located 1.5 -meter depth from the surface, the signal only can be received within 4-meter range of distance between the transmitter node and the receiver node. The rate of received power loss at this point is $3.5 \mathrm{dBm}$ per meter.

\section{CONCLUSION}

As the conclusions, the farthest the signal travel at the surface or the water, the lower the received power that has been received by the receiver. The deep the transmitter sinking vertically, the lower the received power that received by the receiver. The range of the signal travel is different based on the distance travel and depth travel. At the 0.5 meter depth, the signal is able to travel in 12-meter range; farthest range of traveling through the water with small power loss at receiver, $1.6 \mathrm{dBm}$ per meter. At depth of 0.5 meter is the optimum condition for deploying the application of UWSN. It has low of depletion received power per meter. Several numbers of received nodes are needed for deploying the application UWSN at the UTM swimming pool.

\section{ACKNOWLEDGEMENTS}

The authors would like to express the deepest gratitude to the Research Management Center (RMC), Universiti Teknologi Malaysia (UTM) for their support with the Research Student Grant (RSG) number:" VOT Q. J130000.2623.12J66 and to UTM Sport Centre Staff who are allow and provide the safety to the authors to carry out this experiments.

\section{REFERENCES}

[1] R. Khan, S. U. Khan, R. Zaheer and S. Khan, "Future Internet: The Internet of Things Architecture, Possible Applications and Key Challenges," 2012 10th International Conference on Frontiers of Information Technology, Islamabad, 2012, pp. 257-260.

[2] P. P. Ray, "Home Health Hub Internet of Things (H3IoT): An architectural framework for monitoring health of elderly people," 2014 International Conference on Science Engineering and Management Research (ICSEMR), Chennai, 2014, pp. 1-3.

[3] C. Sowmya, C. D. Naidu, R. P. Somineni and D. R. Reddy, "Implementation of Wireless Sensor Network for Real Time Overhead Tank Water Quality Monitoring," 2017 IEEE 7th International Advance Computing Conference (IACC), Hyderabad, 2017, pp. 546-551.

[4] X. Liu, J. Cao, S. Lai, C. Yang, H. Wu and Y. L. Xu, "Energy efficient clustering for WSN-based structural health monitoring," 2011 Proceedings IEEE INFOCOM, Shanghai, 2011, pp. 2768-2776.

[5] Qureshi, Umair Mujtaba, et al. "Rf path and absorption loss estimation for underwater wireless sensor networks in different water environments", Sensors, vol. 16, no. 6, pp. 890, 2016

[6] Chitre, Mandar, Shiraz Shahabudeen, and Milica Stojanovic. "Underwater acoustic communications and networking: Recent advances and future challenges", Marine technology society journal, vol. 42, no. 1, pp. 103-116, 2008.

[7] J. Krautkramer and H. Krautkramer, "Ultrasonic Testing by Determination of Material Properties", Ultrasonic Testing by Determination of Material Properties, pp. 528-550, 1990.

[8] I. Vasilescu, K. Kotay, D. Rus, M. Dundabin, and P. Corke, "Data collection, storage, and retrieval with an underwater sensor network", Proceedings of the 3rd international conference on Embedded networked sensor systems-SenSys, vol. 05, pp. 154, 2005.

[9] Stojanovic, Milica, and Pierre-Philippe J. Beaujean. "Acoustic communication", Springer Handbook of Ocean Engineering, pp. 359-386, 2016.

[10] Lanbo, Liu, Zhou Shengli, and Cui Jun-Hong. "Prospects and problems of wireless communication for underwater sensor networks", Wireless Communications and Mobile Computing, vol. 8, no. 8, 2008.

[11] \X. Che, I. Wells, P. Kear, G. Dickers, X. Gong and M. Rhodes, "A Static Multi-hop Underwater Wireless Sensor Network Using RF Electromagnetic Communications," 2009 29th IEEE International Conference on Distributed Computing Systems Workshops, Montreal, QC, 2009, pp. 460-463.

[12] Rhodes, Mark. "Electromagnetic Propagation in Sea Water and its value in Military Systems", SEAS DTC Technical Conference. 2007.

[13] X. Che, I. Wells, G. Dickers, P. Kear and X. Gong, "Re-evaluation of RF electromagnetic communication in underwater sensor networks," in IEEE Communications Magazine, vol. 48, no. 12, pp. 143-151, December 2010.

[14] Sitter, N. J., et al. "Mussel-based biosensing for hydrologic and eco-biologic processes", AGU Fall Meeting Abstracts. 2009. 
[15] Hattab, Ghaith, et al. "An underwater wireless sensor network with realistic radio frequency path loss model", International Journal of Distributed Sensor Networks, vol. 9, no. 3, 2013:

[16] Module User V1.18, HC-12 Wireless Serial Port Communication, Elecrow, 2012.

[17] A. A. Abdou, A. Shaw, A. Mason, A. Al-Shamma'a, J. Cullen and S. Wylie, "Electromagnetic (EM) wave propagation for the development of an underwater Wireless Sensor Network (WSN)," SENSORS, 2011 IEEE, Limerick, 2011, pp. 1571-1574.

[18] G. Stuber, "Principles of Mobile Communication", Klumer Academic Publishers, 2001.

[19] U. Chakraborty, T. Tewary and R. P. Chatterjee, "Exploiting the loss-frequency relationship using RF communication in Underwater communication networks," 2009 4th International Conference on Computers and Devices for Communication (CODEC), Kolkata, 2009, pp. 1-4.

[20] F. Yunus, S. H. S. Ariffin and Y. Zahedi, "A Survey of Existing Medium Access Control (MAC) for Underwater Wireless Sensor Network (UWSN)," 2010 Fourth Asia International Conference on Mathematical/Analytical Modelling and Computer Simulation, Bornea, 2010, pp. 544-549.

[21] Atmel, "8-bit AVR Microcontroller", ATmega48/V / 88/V / 168/V datasheet, 2016.

[22] Silicon Labs, "High-Performance, Low-Current Transceiver", Si4464/63/61/60 datasheet, 2016 [Revision 1.2].

[23] Keysight Technologies, "Handheld Spectrum Analyzer (HSA)", N9342C 9\& GHz0 datasheet, December 1, 2017.

[24] Great Scott Gadgets, “ANT500”, Available at: https://greatscottgadgets.com/ant500/ [Accessed 11 Feb. 2019].

[25] Spectrum Analyzer Mode Measurement Guide, Agilent X-Series Signal Analyzer, Agilent Technologies, 2010.

\section{BIOGRAPHIES OF AUTHORS}

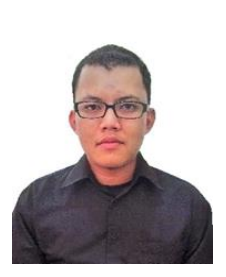

Muhammad Ramdhan M.S received the B.S. degree in Electrical-Electronic Engineering from Universiti Teknologi Malaysia in 2016 and he is currently enrolled in the M.S. degree in Faculty of Electrical Engineering at Universiti Teknologi Malaysia. His current research interests are in the area of wireless networks including wireless sensor networks (WSN), sports and safety monitoring engineering.

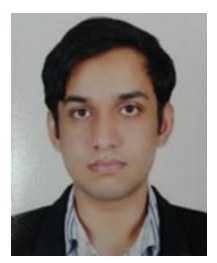

Muhammad Ali completed his Master of Engineering (Electronics and Telecommunications) from Universiti Teknologi Malaysia in 2016. He received his Bachelors of Science in Electrical Engineering from the University of Lahore Pakistan. He is currently doing a Ph.D. in the department of electrical engineering in Universiti Teknologi Malaysia. His topics of interest are big data analytics, machine learning, Internet of Things (IoT), image processing and signal processing.

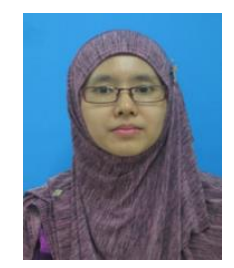

Nurzal Effiyana G received the B.E. (2007) degree from Universiti Teknologi Malaysia. She received two M.E. degree, which is from the Shibaura Institute of Technology (2010) and Universiti Teknologi Malaysia (2011). She received Ph.D. (2016) from Universiti Teknologi Malaysia. Now she is working in Faculty of Electrical Engineering at Universiti Teknologi Malaysia as a senior lecturer who is currently active in research of Mobility Management, Sports Monitoring System, Ubiquitous Network, $5 \mathrm{G}$ and Handover Management.

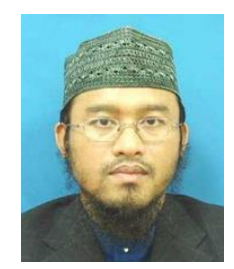

Samura Ali received the Bachelor of Engineering (BE) in Electrical, Electronics and Communications Engineering (1989) from Universiti Teknologi Malaysia. He received a Master's Degree (1998) from the University of Bradford in Electrical, Electronics and Communications Engineering. Currently, now he is working in the Faculty of Electrical Engineering at Universiti Teknologi Malaysia as a senior lecturer.

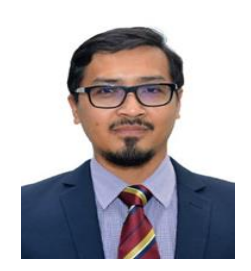

Kamaludin M.Y received the B.Eng degree in Electrical-Electronics Engineering and M.Eng degree in Electrical Engineering from Universiti Teknologi Malaysia. He received a Ph.D. degree from the University of Essex, U.K. He is currently a senior lecturer in the Department of Communication Engineering, Faculty of Electrical Engineering and member of Advanced Telecommunication Technology at Universiti Teknologi Malaysia. His current research interests include Internet-ofThings, Big Data and Software-Defined Networks. 